Article available at http://www.parasite-journal.org or http://dx.dol.org/10.1051/parasite/200108s207

\title{
Highlights of the tenth International Conference ON TRICHINELLOSIS: PAST AND FUTURE
}

\author{
DUPOUY-CAMET J.*, BOIREAU P.** \& MURRELL K.D.***
}

Summary :

The Tenth International Conference on trichinellosis, organized by the International Commission on Trichinellosis, gathered together 147 scientists from 34 countries. The 173 presentations ranged over most aspects of this parasitic zoonosis: history, epidemiology, speciation, biology, immunology, biochemistry, human and animal pathology, new diagnostic methods and control.

KEY WORDS : Trichinella, trichinellosis.

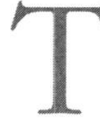

The Tenth International Conference on trichinellosis, organized by the International Commission on Trichinellosis, was convened in Fontainebleau in August 2000 and gathered together 147 scientists from 34 countries. The 173 presentations ranged over most aspects of this parasitic zoonosis: history, epidemiology, speciation, biology, immunology, biochemistry, human and animal pathology, new diagnostic methods and control. A major theme of this Conference was the accomplishments in Trichinella research over the past 150 years and their relevance to the needs and issues in the $21^{\text {st }}$ century.

During the plenary lectures, William C. Campbell (Drew University) reviewed the history of research on Trichinella from the middle of the nineteenth century up to the last war alternatively using pictures of the principal parasitologists or events, and drawings of the first graphic representations of Trichinella. This revealed that many of the difficulties encountered in the early days persist into the $21^{\text {st }}$ century. Jean Blancou (OIE) reminded us that the surveillance and control of trichinellosis in pigs began in Germany as early as 1863 underlining with K. Darwin Murrell (President of the International Commission on Trichinellosis) that the past difficulties were both technical and political and that the persistence of trichinellosis (and its reemergence in many regions) are also linked to

\footnotetext{
* R. Descartes University, EA 2499/INSERM U529, Hôpital Cochin, 27, Fbg St-Jacques, 75014 Paris, France.

** UMR BIPAR, INRA AFSSA ENVA, 22, rue P. Curie, 94703 MaisonsAlfort, France.

*** Danish Centre for Experimental Parasitology, Ridebanevej 3, 1870 Frederiksberg C, Copenhagen, Denmark.
}

changes in economic and political conditions, changes in agricultural practices and human behavioral shifts. The growing importance of sylvatic species was also emphasized.

The reports from the systematics session were very informative. The knowledge of the parasite's taxonomy has progressed greatly in the past 10 years and more than one thousand isolates are now kept in the International Trichinella Reference Center in Rome. This valuable resource has facilitated a rapid change in the taxonomy of Trichinella. Currently, 10 genotypes of Trichinella have been described and six of them are considered as valid species. In addition to $T$. spiralis, T. nativa, T. britovi, T. nelsoni, and T. pseudospiralis, two new species have recently been described, T. murrelli and $T$. papuae. Trichinella murrelli is found in wild animals from temperate regions of North America and T. papuae in feral pigs from Papua New Guinea. This last species shares the characteristic with $T$. pseudospiralis of being non-encapsulated in muscles but its size is similar to that of T. spiralis (Pozio et al.). Edoardo Pozio proposed establishing a new genus, Acystis, to contain these two non-encapsulated species. In an important advance, it is now possible to differentiate all genotypes of Trichinella, using a single muscle larva, by single or multiplex PCRs with primers derived from internal transcribed spacers, micro-satellites or rDNA (Zarlenga et al.; Van der Giessen et al.). Michel Tibayrenc (IRD) reported his experience from research on Trypanosoma cruzi and emphasized that the tools used to characterize species and populations in phylogenetic studies should be adapted to the reliable molecular clock (the fast markers) and that data obtained on Trypanosoma could be useful for the study of Trichinella speciation.

In sessions on the epidemiology of this zoonosis, it is clear that human trichinellosis is emerging or reemerging in some countries. Impressive data were presented for Rumania (more than 15,000 human cases from pork in the past 10 years, Curca et al.), China (around 25,0000 cases from pork, sheep or dog in the past 35 years, Wang \& Cui; Liu \& Boireau), Argentina (more than 5,200 cases from pork in the past ten years, 
Bolpe \& Boffi). The situation is particularly troublesome in countries where social and political upheavals have occurred in the past few years, such as Yugoslavia (Cuperlovic et al.), Croatia (Marinculic et al.), Lithuania (Laiskonis et al.). However, trichinellosis remains a problem even in countries with high economical standards, despite costly veterinary control. In France (Haeghebaert et al.) and Italy (Tamburrini et al.), outbreaks involving several hundreds of patients were linked to horse meat imported from Eastern European countries, whereas in Germany two outbreaks were related to domestic pork sausages (Rehmet et al.; Noeckler et al.). A human outbreak of 336 patients was reported in the Slovak republic after consumption of dog meat sausages during a traditional festival (Dubinski et al.). Thierry Ancelle \& Jean Dupouy-Camet described an active system for surveillance of human cases recently implemented in France. In contrast, other reports revealed that the prevalence of Trichinella in Poland has been decreasing in five years in swine (a 8 -fold reduction) and in wild boars (a 2-fold reduction); this reduction coincided with a diminution of the number of human cases (Ramisz et al.). Epidemiological studies on wildlife were also presented. For example, in Finland, four Trichinella species (T. spiralis, T. nativa, $T$. britovi and T. pseudospiralis) coexist in the same biotope and that raccoon dogs may host four species (Oivanen et al.).

The biology of the parasite remains a fascinating topic of research for many groups. Bernadette Connolly reported on the expression of developmentally regulated genes in capsulated and non encapsulated species of Trichinella. Judith Appleton et al. demonstrated that her group could obtain Trichinella adults and new born larvae release in Caco-2 epithelial cell culture; this innovation is a major advance and will no doubt be very useful for biological and immunological studies or for the screening of anthelminthics compounds. New insights on nurse cell biology and formation were presented. The role of satellite myogenic stem cells in encapsulated and non encapsulated species was studied (Takahashi et al.). The induction of an oxidative stress by the larva in the nurse cell was demonstrated (Bruschi et al.). Endonuclease activities of ES products of the larva were suggested to maintain the reorganized infected muscle cell (Mak \& Ko). High RNA polymerase levels were observed in nuclei of infected cells and polymerase was depleted by mebendazole (Yao $\&$ Jasmer). Genes and their expressive products which may be involved in the biology of the parasite were also characterized: thymidilate synthase (Dobrowska et al.), zinc finger homeodomain protein (Kuratli et al.; Garcia-Reyna et al.), genes differentially expressed by heat shock treatment (Wu et al.), serine protease (Trap et al.), metallo-protease (Lun et al.), Rab4 GTP binding protein (Rodriguez et al.). The great advances in general molecular biology are increasingly being exploited to investigate the complex biology of this parasite.

Clinical aspects of trichinellosis continue to be a major interest, and these investigations have, ironically, been facilitated by the increase in outbreaks, which have provided greater opportunities for clinical investigations. The pathology, the diagnosis and the treatment of human trichinellosis is primary a concern for physicians. Wanda Kocieka demonstrated that neurological and electro-physiological disturbances could last for years after infection, raising the issue of what are the chronic sequels of the disease and the difficulties in treating them. A large study on 389 patients showed a better efficacy for albendazole compared to mebendazole (Cretu et al.). In mice, albendazole-cyclodextrin complexes increased the bio-availability and anthelmintic activity of the drug (Garcia-Rodrigues et al.). Improvements in the serological diagnosis of trichinellosis in humans was achieved using the tyvelose synthetic antigen (Bruschi et al.) or the manufactured "Trichinella western blot IgG" kit to eliminate false positive reactions (Andiva et al.).

There has been great progress in the immunology of trichinellosis. The antigens of the parasite are a particular object of study in order to improve the knowledge of the immune pathogenesis of the disease and to refine serological diagnosis. A strong IgA response against new born larvae antigens was characteristic of the acute phase of the disease in humans but was not found during the chronic stages (Mendez-Loredo et al.) Pascal Boireau presented data showing that some antigens were stage and species specific and with several monoclonal antibodies it was possible to follow a cell lineage from the new born larvae stage to adult. The first discovery in a nematode of a semaphorin-like protein was reported; this trans-membrane and secreted protein could play an important role in the immunological modulation and vascular morphogenesis of the nurse cell (Romaris et al.). Glycosylated epitopes of the muscle larvae also appear to be involved in the human serological response (Malmassari et al.). Interestingly, the Trichinella tyvelose antigen was also found in the embryonated eggs of Haemonchus, Trichuris, Toxocara, Ascaris but not in the adult stages of these species (Dea-Ayuela et al.). A number of papers pointed out the importance of the immune response at the early stages of the disease and emphasized the importance of new born larvae antigens. Dante Zarlenga et al. showed that a cloned new born larvae antigen was recognized by infected swine sera. Several other new born larvae specific genes are under characterization (Zarlenga et al.; Liu Mingyuan et al.). The antigenic variability of muscle larvae stage was demonstrated by sequencing the gp53 gene encoding major antigens of 
TSL1 group in several Trichinella species; additional $\mathrm{N}$ glycosylation sites could be determined in $T$. britovi in comparison with $T$. spiralis (Rodriguez et al.). In a model of intestinal explants, secreted antibodies could recognize a restricted pattern of antigens characterizing the three major stages of the parasite: muscle larvae, adults, new born larvae (Garcia-Reyna et al.). New genes coding for other selected antigens were also reported: a $49 \mathrm{kDa}$ highly conserved antigen in encapsulated species (La Rosa et al.) and 34, 37 and $46 \mathrm{kDa}$ antigens (Yuhe Yan et al.).

The cellular aspects of the immunological response were also described: Naohiro Watanabe reported on the gene control of IgE and its role in protection; direct induction of IL4, IL2 and TNF by Trichinella antigens of rat mast cells was described (Arizmendi-Puga et al.), along with lymph node and Peyer's patch involvement in the Th2 response (Korenaga \& Hashiguchi). Derek Wakelin described the different intestinal cells involved in the mucosal and immune response to Trichinella, particularly the function of the Paneth intestinal epithelial cells which may participate in parasite expulsion by the secretion of anti-microbial peptides. Stella Venturiello showed that in rats, pregnancy reinforced the immune response, resulting in an increased killing of larvae. Raffaella Mele et al. presented evidence that in humans infected with $T$. britovi, lymphocytes express CD8, CD 45, the alpha form of the TCR, IL5 and IL10. Experimental models of trichinellosis are of utmost importance for investigating the pathogenesis of the disease and biological characteristics such as host specificity. Christian Kapel et al. infected pigs with nine different genotypes of Trichinella and reported that only T. spiralis, T. britovi and T. nelsoni were able to survive more than 40 weeks in pigs. Vertical transmission was studied in different animal models: fetal transmission was not observed in the pig and fox but was possible in guinea pigs, ferrets and mice though the number of larvae found in fetal tissues were very low (Webster et al.). Experiments concerning larval survival in decaying meat revealed $T$. spiralis could survive for more than three months in pork buried underground (Jovic et al.) and its resistance was increased in correlation with the age of infection (reflecting the thickness of the capsule) (Von Köller et al.). Experimental infections were carried out in ostriches showing that this bird was receptive to T. pseudospiralis and, surprisingly, to T. spiralis (Piergili-Fioretti et al.).

The prevention of trichinellosis rests on individual actions (sufficient cooking or freezing) and on a veterinary control which is based on the direct detection of larvae in the muscles. Various alternative techniques for detecting muscle larvae were discussed: fast serological methods (Sofronic et al:; Viveros et al.; Patrascu et al.; Yuhe Yan et al.); detection of copro-antigens (Boulos et al.); and PCR (Viveros et al.). But the critical requirements are quality assurance for veterinary control including validated tests, written protocols, control of critical points, certified personnel, proficiency testing, accreditation (Gajadhar et al.) and good production practices for reducing the risk of exposure of pigs to Trichinella (Gamble et al.).

Finally, this conference reminded us that this parasite's unique biological features serve well in helping it to persist in the face of strenuous and costly efforts to eliminate it from the food chain. Because of Trichinella's reemergence as an important public health risk, and the consequent stimulation of research, our knowledge of the parasite has grown. From this research, a greater insight into the complexity of Trichinella biology and epidemiology has been gained, including a realization that although we probably will eventually succeed in eliminating the domestic cycle, the continued existence of Trichinella as a potential zoonosis is likely. This conference highlighted the major features of this parasite's adaptive traits and the human factors that account for its reemergence in many regions, along with the actions of the International Trichinellosis Commission (home page: http://www.med.unipi.it/ict/welcome.htm) to address these issues. The attractiveness of Trichinella as a research subject for study of the parasitic lifestyle and host-pathogens interactions will certainly continue to stimulate the interest of scientists, physicians and all individuals involved in public health and food safety.

\section{ACKNOWLEDGEMENTS}

TCT10 was organised by the International Commission on Trichinellosis and la Société Française de Parasitologie. ICT10 was supported by Université René Descartes (Paris), AFSSA (Maisons-Alfort), INRA, École Nationale Vétérinaire d' Alfort, Assistance PubliqueHôpitaux de Paris, Institut de la Veille Sanitaire, Office International des Epizooties, Ministère de l'Éducation Nationale, Mairie de Fontainebleau, INSERM, USDA. Sponsorship was given by Smith Kline Beecham (UK) and Merial (France). 